\title{
P) Científic@ \\ AVALIAÇÃo FÍSICO-QUÍMICA E MICROBIOLÓGICA DE DOCE DE EXTRATO DE SOJA COM DIFERENTES ADOÇANTES
}

\author{
PHYSICOCHEMICAL AND MICROBIOLOGICAL EVALUATION OF SOYBEAN EXTRACT DULCE \\ WITH DIFFERENT SWEETENERS
}

\author{
Marcio Ramatiz Lima Santos ${ }^{1}$, Natália Oliveira Silva ${ }^{2}$
}

\begin{abstract}
${ }^{1}$ Autor para correspondência; Professor do Instituto Federal Goiano Ceres desde 1995. Licenciado em Ciências Agrícolas pela UFRRJ, Mestre em Ciência e Tecnologia de Alimentos pela URFFJ, Doutor em Energia Nuclear na Agricultura e Meio Ambiente pela ESALQ/USP. Professor do IF Goiano desde 1995.

${ }^{2}$ Estudante do Curso de Bacharelado em Agronomia do Instituto Federal Goiano Campus Ceres, Ceres-GO, Brasil
\end{abstract}

\section{Info}

Recebido: $12 / 2019$

Publicado: 04/2020

DOI: 10.29247/2358-260X.2020v7i1.4114

ISSN: 2358-260X

Palavras-Chave

alimento funcional; alimento saudável;

açúcares, lactose

Keywords:

functional food, healthy food, sweeteners, lactose

\section{Resumo}

O doce de leite é um produto resultante da cocção do leite com açúcar até a concentração e caramelização desejada, cuja produção no Brasil encontra-se ao redor de 34.000 t/ano. 0 objetivo deste trabalho foi avaliar as propriedades físicoquímicas (umidade, cinzas, $\mathrm{pH}$ e acidez titulável) e microbiológicas (Coliforme total e termotolerante) de doce de extrato de soja com diferentes adoçantes. Seis formulações de doce de extrato de soja com diferentes adoçantes foram preparadas: industrializado com açúcar cristal (LN), extrato industrializado com açúcar demerara (LD), extrato industrializado com açúcar mascavo (LM), extrato caseiro com açúcar cristal (SN), extrato caseiro com açúcar demerara (SD) e extrato caseiro com açúcar mascavo (SM). Os resultados das análises foram tabulados e submetidos a ANOVA e ao Teste de Tukey ao nível de 5\% usando o software ASSISTAT. Todas as amostras apresentaram contaminação para coliforme total após 48 horas de incubação a $35^{\circ}$, as amostra $\mathrm{LM} \mathrm{e} \mathrm{SN}$ apresentaram maior contaminação (43 NMP.g- ${ }^{1}$ ). Na análise de acidez titulável houve diferença estatisticamente entre as amostras LN e LM, respectivamente 0,27 e 0,18 \% m/v. Para a análise de pH, todas as amostras diferiram entre si, sendo SD maior acidez $(6,69)$ e menor acidez SN $(7,78)$. Para a análise de umidade a amostra SN $(31,34 \%)$ se diferiu estatisticamente das amostras SM (11,95\%) e LD (11,21\%) mas não se diferiu das demais amostras. No teste de cinzas não houve diferença estatisticamente entre as amostra SN (0,79\%) e SD (1,00\%), entretanto, as mesma diferiram das demais amostras. Não houve diferença estatística para a análise sensorial, mas recomendase o doce de extrato de soja industrial com açúcar mascavo por estar dentro dos padrões recomendado pela legislação brasileira e ter uma melhor aceitabilidade. Os resultados mostraram que o doce de extrato de soja é uma alternativa tecnológica ao doce de leite comum, e atende aos consumidores que apresentam intolerância a lactose.

\section{Abstract}

Dulce de leche is basically a product made from cooked milk and sugar until achieve the desired concentration and caramelization. The Brazilian dulce de leche production it is around 34,000 ton/year. This work aimed to evaluate the physicochemical (humidity, ash, $\mathrm{pH}$ and titratable acidity) and microbiological (total coliform and thermotolerant coliform) of soybean extract dulce with different sweeteners. Six formulations (Milk+sugar cane MC, milk+ demerara - MD, milk + brown sugar - MB, soybean + sugar cane - SC, soybean + demerara - SD and soybean + brown sugar SB) were prepared. The results were submitted to ANOVA and the Tukey Test at $5 \%$ of significance to verify the interactions between the averages using the ASSISTAT. All samples presented contamination for coliform total after $48 \mathrm{~h}$ of incubation at $35^{\circ} \mathrm{C}$ and the samples MB and SC presented higher contamination (43 MPN.g-1). To Titratable acidity there was statistical difference between MC and MB, $0.27 \% \mathrm{~m} / \mathrm{v}$ and $0.18 \% \mathrm{~m} / \mathrm{v}$ respectively. To $\mathrm{pH}$, all samples differed statistically from each other, being SD the highest acidity (6.69) and the lowest acidity to SC (7.78). To humidity, the sample SC (31.34\%) statistically differed from SB (11.95\%) and MD (11.21\%), but do not differed from the other ones. To ash, there was no statistical difference between SC $(0.79 \%)$, SD $(1.00 \%)$, but they differed from the other ones. There was no difference to sensory evaluation for all samples, but we recommend the sample MB to attending the standard of legislation and for it presenting higher score of acceptance. The results shown that the soybean dulce is a technological alternative to the regular dulce de leche, and attending to intolerant consumers for lactose. 


\section{INTRODUÇÃO}

O doce de leite é um produto típico da América Latina, produzido e consumido em grande escala no Brasil e na Argentina. É basicamente um produto resultante da cocção do leite com açúcar até a concentração e caramelização desejada, cuja produção no Brasil encontra-se ao redor de 34.000 t/ano (MACHADO, 2005). O doce de leite apresenta elevado valor nutricional por conter proteínas e minerais, além do alto conteúdo energético (FEIHRMANN; CICHOSKI; REZENDE, 2004.

Ultimamente, o interesse da população é consumir alimentos mais saudáveis (MACHADO, 2012). Juntamente com os novos conceitos de dieta e saúde, a indústria alimentícia vem investindo muito no aprimoramento do "leite/extrato de soja", disponibilizando no mercado produtos de excelente qualidade, despertando, assim, as atenções do mercado mundial de bebidas (VENTURINI et al., 2010).

É um grande desafio o desenvolvimento de novos produtos alimentícios funcionais, que ao mesmo tempo sejam atrativos ao paladar do consumidor e atendam a demanda por produtos saudáveis (MARCHIORI e NAVARINI, 2012).

O leite/extrato de soja é obtido pelo processo de trituração dos grãos de soja, não apresenta sabor ou odor desagradável (DELLA et al., 2003). Um alimento de baixo custo, de alto valor nutritivo e de fácil obtenção (SANTOS et al., 2010). Segundo Torrezan et. al (2003), é de conhecimento geral que o grande obstáculo para o maior consumo deste produto está relacionado ao seu sabor acentuado e característico.

De acordo com Messa et al. (2017) é de grande importância a produção de açúcar para a economia brasileira, a estimativa para 2018 era que a produção chegasse até 40 milhões de tonelada de açúcar, uma estimativa maior que o ano de. Segundo (ULIANA, 2009) o processo da obtenção do açúcar inclui a extração do caldo da cana, clarificação e concentração do mesmo. Existem dois tipos de açúcares que são considerados os mais fabricados, que são eles: demerara e cristal branco (BEHRNS; SILVA, 2004).

A presente pesquisa teve como objetivo avaliar as propriedades físico-químicas e microbiológicas de doce de extrato de soja com diferentes adoçantes.

\section{MATERIAL E MÉTODOS}

Seis formulações do doce foram preparadas, sendo uma com base em grãos de soja processados em liquidificador e a outra utilizando extrato de soja industrializado e adoçadas com três tipos diferentes de açúcar, as formulações foram: extrato industrializado com açúcar cristal (LN), extrato industrializado com açúcar demerara (LD), extrato industrializado com açúcar mascavo (LM), extrato caseiro com açúcar cristal (SN), extrato caseiro com açúcar demerara (SD) e extrato caseiro com açúcar mascavo (SM).

O primeiro doce foi fabricado na cidade de Nova Glória-GO, de forma caseira. Inicialmente se obteve aproximadamente $3 \mathrm{~kg}$ de soja, utilizando $200 \mathrm{~g}$ do produto para 1 litro de água onde se extraiu cerca de $700 \mathrm{ml}$ de extrato de soja, a mistura foi processada no liquidificador por 10 minutos, filtrado em tecido voal, para retirar a parte grosseira do produto conhecida como okara.

O processo de fabricação do doce foi feito três vezes, uma para cada tipo de açúcar. Em seguida levouse o extrato ou fogo brando, onde se adicionou $200 \mathrm{~g}$ de açúcar cristal, açúcar demerara ou açúcar mascavo; ou seja um tipo de açúcar para cada $700 \mathrm{~mL}$ de extrato produzido; em seguida deixou-se os mesmos em fogo brando por aproximadamente $1 \mathrm{~h}$ e meia.

O segundo foi feito utilizando extrato de soja industrializado, onde retirou-se $700 \mathrm{~mL}$ do produto para fabricar o doce, os $700 \mathrm{~mL}$ foram levados ao fogo brando onde se adicionou os $200 \mathrm{~g}$ do açúcar cristal, 
açúcar demerara ou açúcar mascavo; ou seja um tipo de açúcar para cada $700 \mathrm{~mL}$ de extrato de soja, os mesmos ficaram em fogo brando por aproximadamente $1 \mathrm{~h} \mathrm{e}$ meia. Depois de fabricado o produto foi embalado em recipiente de vidro transparente, levado para o Instituto Federal Goiano - Campus Ceres, para serem realizados as análises microbiológicas e físico-química.

\section{Análise Microbiológicas}

As amostras foram preparadas de acordo com o descrito por Santos e Marques (2010) com modificações, utilizando $1,0 \mathrm{~g}$ de amostra, pesadas em balança analítica e homogeneizadas em 9,0 mL de Peptona estéril e depois transferiu-se $1 \mathrm{~mL}$ desta mistura nos tubos contendo $9,0 \mathrm{~mL}$ de Caldo Lactosado, nas diluições 10-1, 10-2 e 10-3, foram utilizados 54 tubos. Os tubos foram incubados em câmara BOD a pôr 48h. A determinação foi feita pela Técnica de Tubos Múltiplos - NMP (número mais provável). A combinação desses resultados é usada na determinação do NMP/g. Na leitura dos tubos, considerava-se como positivos os que apresentaram-se turvos, com formação de gás nos tubos de Durham invertidos, com isso indicando a presença de organismos coliformes, e os negativos não apresentaram presença de gás no tubo de Durham.

Os tubos positivos foram repicados para tubos contendo continham $9 \mathrm{~mL}$ de caldo verde brilhante com tubo de Durham invertido, e incubados em estufa a $35^{\circ} \mathrm{C}$ para se obter os resultados confirmativos, e outros tubos com $9 \mathrm{~mL}$ de caldo EC com tubos de Durham invertidos e incubados em estufa a $45^{\circ} \mathrm{C}$ para se obter os resultados para coliformes termotolerantes.

\section{Análise Físico-Quimica}

Para as análises de $\mathrm{pH}, 10$ gramas de cada amostra foram pesadas e homogeneizadas em água destilada; enquanto se pesava as amostras o pHmetro era calibrado, as análises foram feitas em triplicatas. Para as análises de acidez titulável 10 gramas de cada amostra foram pesadas e homogeneizadas em água destilada, usou-se solução de $\mathrm{NaOH} 0,1 \%$ para realizar o procedimento, para cada mistura homogeneizada foram adicionados 3 gotas de fenolftaleína.

Para a analises de umidade foram pesadas 5 gramas de cada amostra, pesadas em balança analítica e levadas para a mufla por 2 horas, em seguida as amostras pesadas novamente para se obter os resultados. Em seguidas as amostras foram levadas novamente para a mufla por 48 horas e assim se obter os resultados de cinzas.

\section{Análise Estatística}

Os resultados das análises foram tabulados e submetidos a ANOVA e ao Teste de Tukey ao nível de $5 \%$ usando o software ASSISTAT.

\section{RESULTADOS E DISCUSSÕES}

Na preparação do doce de extrato de soja a sanitização foi realizada de forma rigorosa, para que não houvesse nenhum tipo de contaminação. Na Tabela 1 são apresentados os resultados obtidos após 48 horas de incubação na técnica de tubos múltiplos para determinação de coliformes. Todas as amostras apresentaram contaminação, sendo a maior contaminação observada para as amostras LM e SM com $43 \mathrm{NMP} / \mathrm{g}$ e a menor contaminação foi observada pela amostra $\mathrm{LD}$ com $<3,0 \mathrm{NMP} / \mathrm{g}$. As demais das amostras apresentaram baixa contaminação e se encontravam de acordo com os padrões exigidos pela legislação brasileira.

Santos et al. (2016), avaliaram doce de leite industrializado onde os resultados obtidos para a contagem de coliformes totais em doce de leite em industrializado, indicaram que as amostras de doce leite pastoso estavam no padrão preconizado pela portaria 
354 de 04 de setembro de 1997, encontrando valores baixos como $<3,0$. Assim como nessa pesquisa, onde as amostras SD, LN, LD, SM também apresentaram valores baixos de contaminação.

Tabela 1. Resultados das análises de coliformes totais de doces de extrato de soja com diferentes adoçantes.

\begin{tabular}{cc}
\hline Amostra & $\mathbf{N M P} / \mathbf{g}$ \\
\hline SN & 43 \\
SD & 3,6 \\
SM & 3,6 \\
LN & 9,2 \\
LD & $<3,0$ \\
LM & 43 \\
\hline
\end{tabular}

De acordo com Abreu et al (2007), a presença de coliformes totais em alimentos pode ser indicativo de tratamento térmico inadequado ou de uma provável contaminação posterior, causada no processo de manuseio das próprias análises.

$\mathrm{Na}$ tabela 2 são apresentados os resultados para coliformes termotolerantes, onde observou-se que todas as amostras se apresentavam sem contaminação por coliformes termotolerantes.

Tabela 2. Resultados das análises de coliformes termotolerantes de doces de extrato de soja com diferentes adoçantes.

\begin{tabular}{cc}
\hline Amostra & $\mathbf{N M P} / \mathbf{g}$ \\
\hline SN & $<3,0$ \\
SD & $<3,0$ \\
SM & $<3,0$ \\
LN & $<3,0$ \\
LD & $<3,0$ \\
LM & $<3,0$ \\
\hline
\end{tabular}

A Resolução RDC no 12/2001 (BRASIL, 2001) determina um limite de até $500 \mathrm{NMP} / \mathrm{g}$ de coliformes termotolerantes para doce de leite e de até $100 \mathrm{NMP} / \mathrm{g}$ em alimentos vegetais tipo purês e geleias.

A Tabela 3 apresenta os resultados das análises de $\mathrm{pH}$, umidade, cinzas e acidez titulável dos doces de extrato de soja com diferentes adoçantes.

Tabela 3. Análises físico-químicas dos doces de extrato de soja com diferentes adoçantes.

\begin{tabular}{ccccc}
\hline Amostra & $\mathbf{p H}$ & Umidade & Cinzas & Acidez Titulável \\
\hline SN & $7,78 \pm 0,02 \mathrm{a}$ & $31,34 \mathrm{a}$ & $0,79 \mathrm{~b}$ & $0,21 \pm 0,03 \mathrm{ab}$ \\
SD & $6,69 \pm 0,02 \mathrm{~d}$ & $25,78 \mathrm{ab}$ & $1,00 \mathrm{~b}$ & $0,24 \pm 0,03 \mathrm{ab}$ \\
SM & $6,83 \pm 0,02 \mathrm{c}$ & $11,95 \mathrm{~b}$ & $2,54 \mathrm{a}$ & $0,22 \pm 0,03 \mathrm{ab}$ \\
$\mathrm{LN}$ & $6,70 \pm 0,02 \mathrm{~d}$ & $17,98 \mathrm{ab}$ & $0,88 \mathrm{a}$ & $0,27 \pm 0,03 \mathrm{a}$ \\
$\mathrm{LD}$ & $7,76 \pm 0,02 \mathrm{a}$ & $11,21 \mathrm{~b}$ & $2,73 \mathrm{a}$ & $0,25 \pm 0,03 \mathrm{ab}$ \\
LM & $7,69 \pm 0,02 \mathrm{~b}$ & $16,48 \mathrm{ab}$ & $2,68 \mathrm{a}$ & $0,18 \pm 0,03 \mathrm{~b}$ \\
\hline Média Geral & 7,24 & 19,12 & 1,77 & 0,23 \\
\hline CV $(\%)$ & 0,25 & 30,11 & 14 & 12,58 \\
\hline
\end{tabular}

As médias seguidas pela mesma letra não diferem estatisticamente entre si. Foi aplicado o Teste de Tukey a 5\% de probabilidade.

Oliveira et al (2010) avaliou doces de leite pastoso comercializados a granel no comércio de Lavras/MG, onde também foram avaliados 6 tipos de doces diferentes, os resultados encontrados para $\mathrm{pH}$ foram entre 6,58 até 7,6 . Os resultados chegaram próximos aos valores encontrados para os doces de extratos de soja, que foram valores entre 6,69 até 7,78. Klug (2007), avaliou doces e geleias, e como resultado apresentou que no doce de leite, tradicional ou light, o pH ótimo oscila entre 5,9 e 6,1. Francisquini (2016) chegou-se à conclusão de que o tipo de açúcar influencia no $\mathrm{pH}$ do doce.

Ainda segundo Oliveira et al. (2010), a diferença encontrada na análise de $\mathrm{pH}$ e a acidez titulável das amostras de doce de leite pastoso, pode ser causada pelo processo de fabricação personalizado, pela 
matéria prima e pelos ingredientes que cada indústria utiliza, devido a despadronização do produto entre diferentes marcas. Já para acidez titulável os valores encontrados foram entre 0,27 até 0,31 o que apresentou alta semelhança comparados aos doces de extrato de soja.

Segundo a legislação brasileira (BRASIL, 1997), doces de leite devem conter no máximo 30\% de umidade, para o teste de umidade todas as amostras para o extrato industrializado permaneceram dentro da legislação onde apresentaram valores entre 11,21 e 17,98. Já para o doce de extrato de soja, as amostras SD e SM estavam em conformidade com a legislação, e o doce SN apresentou valor acima de 30\% de umidade.

De acordo com Oliveira (2012), valores acima do recomendado para o teor de umidade pode estar relacionado ao tempo de cocção do produto ou do tipo de adoçante que foi utilizado no processo. Já Carvalho et al., (2014), encontraram teores de umidade inferiores à 26,0\% na produção de doce de leite com teor reduzido de lactose, o que justifica os valores abaixo de 17,98\% encontrados neste estudo.

Francisquini (2016), em seu estudo encontrou umidade, entre $26 \%$ e $40 \%$, os doces pastosos são alimentos produzidos a partir da retirada de água do mesmo através da troca de calor com vapor indireto, através desse processo há uma melhora na palatabilidade e conservação do produto devido à redução no teor de umidade do produto, assim comprovando que teores de umidades abaixo de $26 \%$ são aceitáveis.

Para a análise de cinza o teor máximo permitido pela Resolução 354/1997 do MAPA para doce de leite é de 2,0\%. As amostras SN, SD e LN apresentaram valores permitidos pela legislação, já as amostras SM, LD e LM obtiveram valores acima de 2\% chegando até 2,73. Silvestre et al., (2013) produziram brigadeiros sem lactose utilizando extrato hidrossolúvel de soja e açúcar mascavo na fabricação do mesmo, o brigadeiro obteve um índice de cinzas de 0,5\%, valor perto do encontrado para o doce SN e LN.

(RIBEIRO, 2006) aproveitou resíduo do extrato de soja na elaboração de um produto tipo paçoca e encontrou valores no teor de cinzas, de 1,66\% a 2,66\%. Valores semelhantes ao encontrados para os doces de extrato de soja, assim podendo afirmar que a soja influencia no teor de cinzas dos alimentos. Carvalho \& Berti (2014), encontraram valores médios de cinzas entre 3,65\% e 3,87 \% em doces com $0 \%$ de lactose, o que apresentam superioridade ao estabelecido pela Resolução 354/1997 do MAPA. Portanto, no estudo realizado por Ribeiro et al. (2009) obteve-se $1,64 \%$ no teor de cinzas no doce de leite elaborado com sucralose.

\section{CONCLUSÕES}

Pelo presente trabalho pode-se concluir que o doce de extrato de soja industrializado e/ou caseiro é tecnologicamente viável e pode se tornar um alimento funcional por atender aos consumidores intolerantes à lactose.

Quando se comparou produtos industrializados com os produtos caseiros, pode-se observar diferenças significativas, principalmente quanto ao tipo de matéria-prima utilizada (leite ou extrato de soja).

Nos diferentes tratamentos, observou-se que os parâmetros físico-químicos $(\mathrm{pH}$, acidez titulável, umidade e cinzas) não variaram significativamente com a adição de diferentes para o doce de extrato de soja.

\section{REFERÊNCIAS BIBLIOGRÁFICAS}

ABREU, CRAD, PINHEIRO, AM, MAIA, GA, CARVALHO, JMD, SOUSA, PHMD. Avaliação Química e Físico-Química de Bebidas de Soja com Frutas Tropicais. Araraquara, Jul/Set.de 2007. Disponível em: http://serv- 
bib.fcfar.unesp.br/seer/index.php/ alimentos/article/viewFile/166/174. Acesso em: 02/03/2018.

BRASIL. Ministério da Agricultura, Pecuária e Abastecimento. Portaria $\mathrm{n}^{\circ} 354$, de 4 de setembro de 1997. Acessado em 02/05/2019. Disponível

em: http://www.cda.sp.gov.br/www/legislacoes/p opup.php?action $=$ view\&idleg $=664$

BRASIL, RDC no 12 de 02 de Janeiro de 2001. Regulamento Técnico Sobre Padrões Microbiológicos para Alimentos. 2001.

BEHRNS, JH, SILVA, MAAP. Atitude do Consumidor à Soja e Produtos Derivados. Ciência e Tecnologia de Alimentos. Campinas. 431- 439, jul-set 2004.

CARVALHO, DR, BERTI, MA. Desenvolvimento E Avaliação De Doce De Leite Colonial Light Acrescentado De Aveia Com Calda De Morango. Universidade Tecnológica Federal Do Paraná Coordenação Do Curso De Tecnologia Em Alimentos Curso Superior De Tecnologia Em Alimentos. Francisco Beltrão. 2014.

DELLA, LSM, GOMES, DD, NACHTIGALL, AM, CAVALCANTE, JFM, MINIM, VPR. Perfil Sensorial de doce de leite pastoso. Revista Instituto de Laticínios "Cândido Tostes" set out, no 334, 2003. p. 45-50.

FEIHRMANN, AC, CICHOSKI, AJ; REZENDE, DF. Doce de leite (revisão). Higiene Alimentar, v. 18, n. 118, p.21-23, 2004.

FRANCISQUINI, JA. Caracterização e Avaliação de Indicadores Físico-Químicos, Tecnológicos e de Tratamento Térmico em Doces de Leite. Universidade Federal De Juiz De Fora Programa De Pós-Graduação Em Ciência E Tecnologia Do Leite E Derivados. Juiz de Fora. 2016.

KLUG, TB. Controle de Qualidade em Doces. Universidade Federal De Pelotas Departamento De Ciência Dos Alimentos. Pelotas. Dezembro2007.

MACHADO, L.M.P. Uso de soro de queijo e amido de milho modificado na qualidade do doce de leite pastoso. Faculdade de Engenharia de Alimentos, UNICAMP, Campinas, 2005.
MACHADO, SS. Tecnologia Da Fabricação Do Açúcar. Universidade Federal de Santa Maria. Inhumas: IFG. Santa Maria. 2012.

MARCHIORI, C, NAVARINI, S. Desenvolvimento De Doce De Soja Diet. Francisco Beltrão, 2012.

MESSA, S, NESPOLO, CR. Produção e Composição De Diferentes Tipos De Açúcar. SB Rural. Disponível em:http://www.ceo.udesc.br/arquivos/id_sub menu/285/rural_202.pdf $>$. Acesso em: $09 / 06 / 2018$

OLIVEIRA, ENA. de. Processamento e Caracterização e Armazenamento de Geleia Tradicional e Dietética de Umbu-Cajá. Dissertação. Universidade Federal de Campina Grande. Campina Grande, 2012.

OLIVEIRA, RME, OLIVEIRA, ARC, RIBEIRO, LP, PEREIRA, R, PINTO, SM, ABREU, LR. Caracterização Química De Doces De Leite Comercializados A Granel Em Lavras/Mg. Rev. Inst. Latic. "Cândido Tostes". 2010.

RIBEIRO, NMQ, COSTA, ECM, MORAIS, AS, RENSIS, CMVB. Avaliação das características Físico Químicas e Sensoriais de Doce de Leite Diet Fabricado com Sucralose, Litesse e Lactitol. Ciênc. Biol. Saúde. Londrina, 2009.

RIBEIRO, V. A. Aproveitamento Do Resíduo Do Extrato De Soja Na Elaboração De Um Produto Tipo Paçoca. Universidade Federal de Lavras. 2006.

SANTOS, LF, MENEGHIN, EM, PEDERSOLI, GR, CARNEIRO, B, VALÉRIO, G, RUAS, EA. Análise Microbiológica De Doce De Leite Comercializado $\mathrm{Na}$ Cidade De Novo Itacolomi - PR. Apucurana. 2016.

SANTOS, RA, MARQUES, RCP. Análise Microbiológica E Físico-Química De Doce De Leite Vendido No Comércio Informal De Currais Novos/Rn. Instituto Federal do Rio Grande do Norte. 2010.

SILVESTRE, VR, LAY, VA. Elaboração De Um Doce "Brigadeiro" Isento De Lactose. Universidade Tecnológica Federal Do Paraná Coordenação De Alimentos Curso Superior Em Tecnologia De Alimentos. Ponta Grossa. 2013. 
TORREZAN, R, CECCATO, MC, BARRETO, ACS, SILVA, VS, CARATIN, C, PEREIRA, CG MARTINEZ, J, KUSHIDA, MM, NETO, MP, IAMANAKA, B, CORDEIRO, HMAB. Avaliação do perfil sensorial de alimento com soja sabor laranja. B-CEPPA, Curitiba-PR. 2004.
ULIANA, MR. Bebida Mista de Extrato de Soja e Suco de Amora: Análises

Químicas e Sensorial. Universidade Estadual Paulista "Júlio De Mesquita Filho". Botucatu-SP. 2009.

VENTURINI, FWG. Bebidas não Alcoólicas: Ciência e Tecnologia. Vol. 2 São Paulo. 2010. 